\title{
Real-time functional magnetic resonance imaging in obsessive-compulsive disorder
}

\author{
This article was published in the following Dove Press journal: \\ Neuropsychiatric Disease and Treatment \\ 12 July 2017 \\ Number of times this article has been viewed
}

\section{Óscar F Gonçalves ${ }^{1-3}$ \\ Marcelo C Batistuzzo ${ }^{4}$ \\ João R Sato ${ }^{5}$}

'Neuropsychophysiology Lab, CIPsi, School of Psychology, University of Minho, Braga, Portugal; ${ }^{2}$ Spaulding Neuromodulation Center, Department of Physical Medicine \& Rehabilitation, Spaulding Rehabilitation Hospital and Massachusetts General Hospital, Harvard Medical School, Boston, MA, USA; ${ }^{3}$ Social and Cognitive Neuroscience Laboratory, Center for Health and Biological Sciences, Mackenzie Presbyterian University, ${ }^{4}$ Department and Institute of Psychiatry, University of São Paulo Medical School (FMUSP), ${ }^{5}$ Mathematics, Computing, and Cognition Center, Universidade Federal do $A B C$ - UFABC, São Paulo, Brazil
Correspondence: Óscar F Gonçalves Neuropsychophysiology Lab, School of Psychology, University of Minho, Campus de Gualtar, 47 I0-057 Braga, Portugal

Tel +35I 25360426 I

Fax +35I 253604658

Email goncalves@psi.uminho.pt

\begin{abstract}
The current literature provides substantial evidence of brain alterations associated with obsessive-compulsive disorder (OCD) symptoms (eg, checking, cleaning/decontamination, counting compulsions; harm or sexual, symmetry/exactness obsessions), and emotional problems (eg, defensive/appetitive emotional imbalance, disgust, guilt, shame, and fear learning/extinction) and cognitive impairments associated with this disorder (eg, inhibitory control, working memory, cognitive flexibility). Building on this evidence, new clinical trials can now target specific brain regions/networks. Real-time functional magnetic resonance imaging (rtfMRI) was introduced as a new therapeutic tool for the self-regulation of brain-mind. In this review, we describe initial trials testing the use of rtfMRI to target brain regions associated with specific OCD symptoms (eg, contamination), and other mind-brain processes (eg, cognitive - working memory, inhibitory control, emotional - defensive, appetitive systems, fear reduction through counter-conditioning) found impaired in OCD patients. While this is a novel topic of research, initial evidence shows the promise of using rtfMRI in training the self-regulation of brain regions and mental processes associated with OCD. Additionally, studies with healthy populations have shown that individuals can regulate brain regions associated with cognitive and emotional processes found impaired in OCD. After the initial "proof-of-concept" stage, there is a need to follow up with controlled clinical trials that could test rtfMRI innovative treatments targeting brain regions and networks associated with different OCD symptoms and cognitive-emotional impairments.
\end{abstract}

Keywords: real-time fMRI, obsessive-compulsive disorder, self-regulation, neurofeedback, neuromodulation

\section{Introduction}

Obsessive-compulsive disorder (OCD) remains as one of the most disabling psychiatry disorders. ${ }^{1}$ OCD patients report overwhelming intrusive thoughts, images, ideas, or urges (obsessions - eg, contamination, harm or sexual, symmetry or exactness, and somatic fears), which they attempt to neutralize by repetitive behaviors or mental actions (compulsions - eg, checking, cleaning/decontamination, and counting).

The Diagnostic and Statistical Manual of Mental Disorders - Fifth Edition ${ }^{2}$ has integrated OCD in a new classification designated obsessive-compulsive and related disorders (OCRDs). Likewise, the working group for the eleventh edition of the International Classification of Diseases and Related Health Problems suggested a new category grouping all OCRDs along with body dysmorphic disorder, hoarding disorder, trichotillomania, and excoriation disorder. ${ }^{3}$ Overall, the new classification criteria emphasize inhibitory control deficits (ie, repetitive behaviors and mental actions) rather than problems with emotional regulation (ie, anxiety). Despite this recent emphasis on cognitive impairments, there is widespread evidence that both cognitive and emotional impairments are present in OCD. ${ }^{4}$ OCD is characterized by intense emotional arousal 
and executive control impairments. These two mechanisms influence one another and are responsible for maintaining the obsessive-compulsive cycle. ${ }^{5}$

In this review, we start by summarizing main conclusions of the research looking for mind-brain signatures in OCD. Building on these studies, we then address recent avenues opened by new methodologies aimed at modulating brain regions and circuits using self-regulatory real-time neuroimaging methods. Finally, initial trials testing the use of real-time functional magnetic resonance imaging (rtfMRI) to target brain regions associated with OCD impairments are reviewed.

\section{OCD: mind-brain signatures}

A variety of neuroanatomical models were advanced to explain the pathogenesis of OCD with a common agreement on the existence of dysfunctional cortico-striato-thalamocortical (CSTC) circuitry - a series of loops projecting from the prefrontal cortex (PFC) to the striatum, the thalamus (via the globus pallidus), and back to the cortex. ${ }^{6}$ Despite this consensus, recent studies have been identifying additional brain correlates associated with OCD symptomatology, ${ }^{7}$ helping in generating new hypotheses for the OCD pathogenesis. ${ }^{8,9}$ In what follows, we briefly summarize some of the core findings about the brain's structural and functional signatures of cognitive and emotional impairments present in OCD.

The research on gray matter morphological alterations in OCD endorses the hypothesis of changes in areas associated with the CSTC pathways, particularly dorsolateral prefrontal cortex (dlPFC), orbitofrontal cortex (OFC), anterior cingulate cortex (ACC), thalamus, and striatum. ${ }^{10,11}$ However, there is now evidence for the involvement of regions outside these pathways, such as several regions of the parietal cortex, amygdala, hippocampus, and cerebellum. ${ }^{12}$ For example, a recent MRI volumetric study ${ }^{13}$ confirmed alterations in not only the traditional frontal (decrease of gray matter in the middle frontal gyrus) and subcortical regions (increase of white matter in the pallidum) but also the temporal-parietal regions. These temporal-parietal abnormalities are highly correlated with OCD symptom severity: decrease of gray matter in the superior parietal lobe and white matter in the angular and superior temporal gyri. These morphological abnormalities may help to explain prominent symptoms in OCD, such as difficulties with inhibitory control (pallidum, angular gyrus), executive functioning (middle frontal gyrus), compulsive checking (superior temporal gyrus), and visualspatial deficits (superior parietal lobe). Although this relationship between cognition and morphological abnormalities in OCD was not extensively investigated, initial evidence suggests that brain morphological alterations are associated with not only different OCD symptoms ${ }^{14,15}$ but also specific cognitive deficts ${ }^{16,17}$ observed in OCD.

A recent review of gray matter volumetric and shape alterations in OCD confirmed abnormalities in the following regions: PFC, OFC, thalamus, striatum, and cerebellum (possible associations with impairments in inhibitory control); ACC, insula, amygdala, and supramarginal gyrus (possible association with emotional regulation and fear extinction deficits); dlPFC and hippocampus (possible associations with difficulties in memory monitoring); and superior parietal lobule, occipital lobe, and the precuneus (possible associations with visual-spatial impairments). ${ }^{18}$

Consistent with morphological alterations, there is now evidence for structural connectivity abnormalities in major white matter tracts: superior longitudinal fasciculus (memory, inhibitory control), inferior fronto-occipital fasciculus (inhibitory control), inferior longitudinal fasciculus (emotional regulation, visual-spatial processing), cingulum bundle (inhibitory control, emotional regulation), internal capsule (inhibitory control); different regions of the corpus callosum (inhibitory control, emotional regulation, visual spatial processing), optic radiation (visual-spatial processing), and uncinate fasciculus (memory). ${ }^{19,20}$ For example, recently, we showed connectivity alterations in core regions (ie, different parcellations of the inferior frontal gyrus [IFG]) associated with different components of inhibitory control as evidenced by low levels of fractional anisotropy and high levels of diffusivity in OCD patients. ${ }^{21}$

More important are the results of functional studies in OCD showing abnormal brain activity during rest conditions as well as during cognitive/executive (working memory, cognitive flexibility, and inhibitory control) and emotional tasks (fear/defensive, disgust, guilt, shame). ${ }^{5}$ For example, a dissociation pattern was found between the activity of the defensive and appetitive survival systems in OCD, by showing that patients react to a basic threat with a strong activation of the defensive system mobilizing many of the brain network systems. In an opposite way, activation of the appetitive system is blocked when facing positive emotional triggers (eg, erotic images) from the initial stages of visual processing. ${ }^{22}$ Consistent with this finding, a more recent study showed that OCD patients had difficulties in the deactivation of the default mode network (self-referential processes) even when the non-rest condition included the presentation of provoking emotional stimuli, particularly evident in the case of images with pleasant content. ${ }^{23}$ Additionally, recent studies 


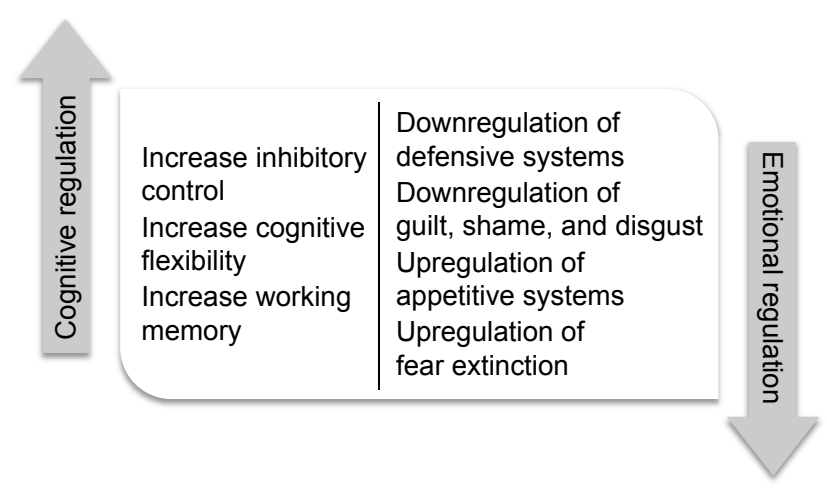

Figure I Objectives for clinical intervention based on findings from neuroimaging research on emotional and cognitive OCD impairments.

Abbreviation: OCD, obsessive-compulsive disorder.

have shown that OCD patients present impairments in fearrelated circuits, particularly illustrated by deficits in extinction retention (ie, patients can extinguish fear-acquired behaviors, but failed to retain this extinction over the time). ${ }^{24,25}$

Summing up, evidence from neuroimaging studies is helping to understand OCD as both an emotional (ie, anxiety and fear-related processes) and a cognitive (ie, inhibitory control) disorder, with a diversity of psychological and symptomatic expressions. But above all, distinct specific cognitive (eg, inhibitory control, working memory, cognitive flexibility) or emotional impairments (eg, affective-motivational imbalance, fear extinction retention) can now be traced to specific brain signatures. Figure 1 illustrates the objectives for intervention based on current findings from neuroimaging research on emotional ${ }^{22,26}$ and cognitive regulation. ${ }^{27-29}$

\section{Brain self-regulation with rtfMRI}

For more than a century, a variety of clinical interventions were used to regulate brain functioning. These methods can be differentiated dependent on the degree of invasiveness (psychological versus surgical) and regulation strategy (external versus self-regulatory). As illustrated in Figure 2, in opposite quadrants are deep brain stimulation ${ }^{30}$ (DBS, ie, a method for regulating brain functioning by implanting, deep in the brain, a stimulation device) and self-regulatory neuromodulation (aka as neurofeedback - a technique by which individuals learn how to regulate a specific brain response by noninvasive real-time feedback of brain activity). Between these two extremes, and occupying different positions in the four quadrants, we have electroconvulsive therapy, ${ }^{31}$ transcranial magnetic stimulation, ${ }^{32}$ transcranial direct current stimulation, ${ }^{33}$ psychopharmacology, and psychotherapy. ${ }^{34}$

Self-regulatory neuromodulation (SRN) refers to a diversity of techniques with the aim of modulating brain activity

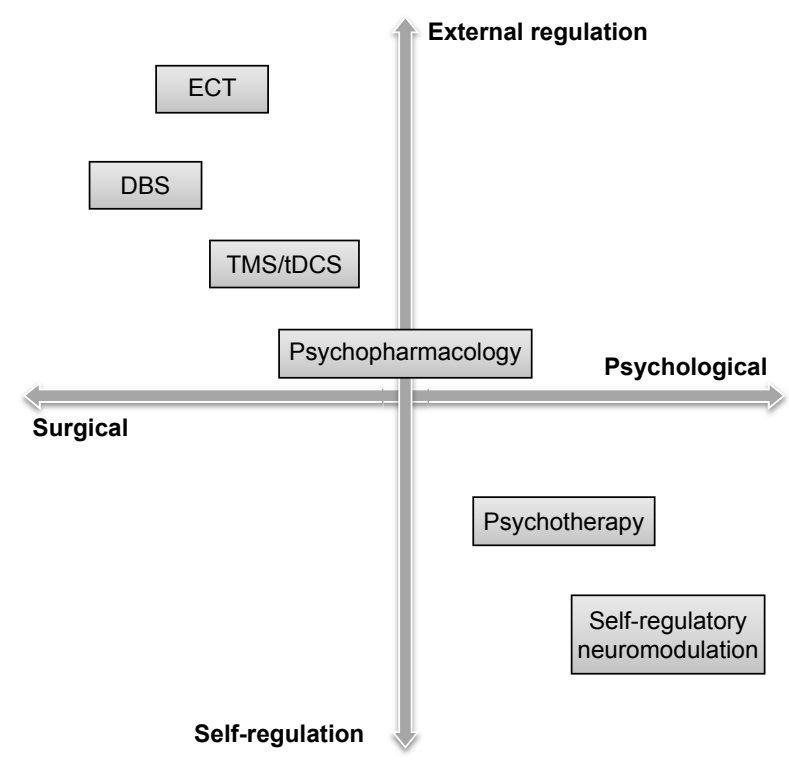

Figure 2 Examples of brain regulation strategies along two axes depending on the degree of invasiveness (psychological versus surgical) and regulation strategy (external versus self-regulatory).

Abbreviations: ECT, electroconvulsive therapy; DBS, deep brain stimulation; TMS, transcranial magnetic stimulation; tDCS, transcranial direct current stimulation.

by having the individual monitoring real-time signals of brain activity and learning how to self-regulate this activity by mechanisms of associative learning. ${ }^{35} \mathrm{SRN}$ was established as a way of modulating electrical neural activity while taking advantage of the cost-effectiveness and high temporal (time) resolution (ie, it can show the time at which changes are occurring in the brain) of the electroencephalogram (EEG). Building on the groundbreaking work done in the late 1960 s by an outstanding cohort of researchers, it was shown that animals and humans could be trained in regulating the brain activity. ${ }^{36}$

We can differentiate SRN methods in terms of both signal resolution (time and spatial) and ecological context (laboratory versus natural environment) (Figure 3). Some signals, such as the EEG, have a quick temporal resolution and can be collected in natural environments. Changes in the brain's electrical activity are associated in time with the brain's response to internal/external events. However, EEG signals, as captured from scalp sensors, are the product of a distributed synaptic activity from multiple brain sources and thus lack information about which brain regions or networks are active. The measurement at each EEG channel is not specific from the brain region below the scalp electrode. This is why we refer to EEG as having a high temporal but a low spatial resolution.

To overcome these limitations, low-resolution electromagnetic tomography (eg, LORETA, sLORETA, eLORETA) 


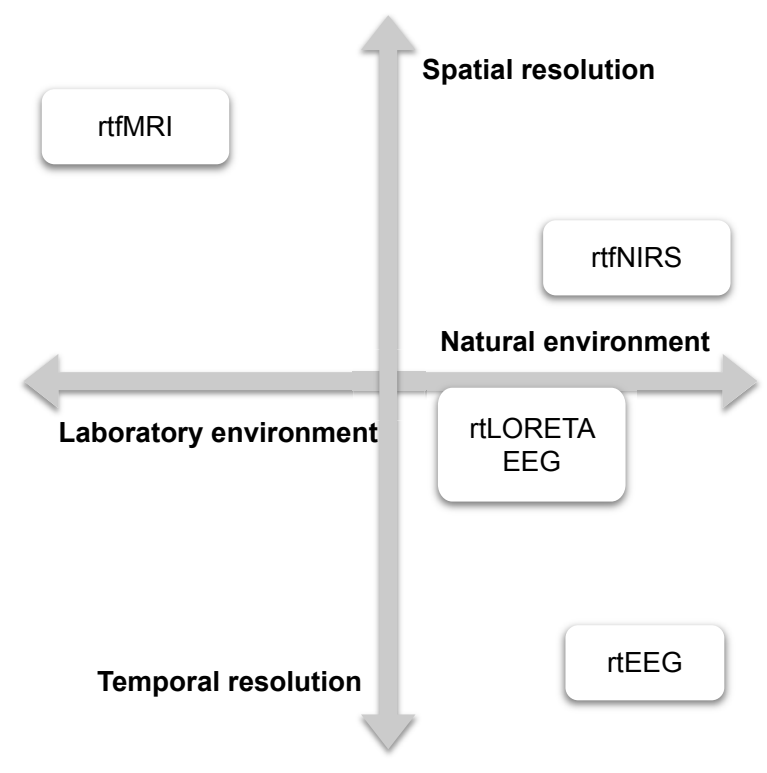

Figure 3 Examples of brain self-regulation strategies along two axes depending on degree signal resolution (time versus spatial resolution) and ecological context (laboratory versus natural environment).

Abbreviations: rtfMRI, real-time functional magnetic resonance imaging; rtfNIRS, real-time functional near-infrared spectroscopy; rtLORETA EEG, real-time lowresolution electromagnetic tomography with electroencephalography; rtEEG, realtime electroencephalography.

was introduced with the main concern in providing estimates of the spatial location of the dipole sources captured by the EEG signals. ${ }^{37}$ Several studies have been applying LORETA rtEEG as a way of moving, from the brain's overall EEG rhythms to specific brain regions in both healthy ${ }^{38}$ and clinical populations (eg, OCD). ${ }^{39}$

Recently, local brain's hemodynamic response (ie, changes in blood flow and oxygenation related to neuronal activity) was selected as the target for SRN. Under certain conditions, the regional blood oxygenation level-dependent (BOLD) signal, measured in $\mathrm{fMRI}$, is considered an indirect measure of local neuronal activity. ${ }^{40}$ Markers of this blood flow are reliable and accurate indices of local activation. In fMRI, the minimum unit of spatial resolution is the "voxel", which has a width, length, and thickness (analogous to the pixel in two-dimensional pictures, but in three-dimensional space). The analysis of the hemodynamic response in a voxelby-voxel fashion enables significant improvements in spatial resolution, typically on the order of less than $3 \mathrm{~mm}$. Since the BOLD signal is based on hemodynamic processes, the changes are slow in time, resulting in a time lag of several seconds between the local activation (triggered by an internal or external event) and the associated change in blood flow. Contrary to rtEEG, rtfMRI provides high spatial but low temporal resolution. Currently, two techniques use the hemodynamic response in SRN: real-time functional nearinfrared spectroscopy (rtfNIRS) and rtfMRI. ${ }^{41}$
rtfNIRS uses the measurement of light absorption in the near-infrared wavelength to quantify oxygenated and deoxygenated hemoglobin in cortical regions. This is a lowcost technique (compared to MRI) with a reasonable high space/time resolution ratio. Difficulties in mapping subcortical regions limit the scope of rtfNIRS for SRN, but several studies are using rtfNIRS to modulate the regions of the frontal cortex, particularly those associated with motor behavior. ${ }^{42}$

rtfMRI was introduced as a process for training the selfregulation of brain-mind processes using the BOLD response as a proxy for brain response. As shown in Figure 4, rtfMRI requires a complex technological procedure that includes: 1) brain signal acquisition inside an MRI scanner; 2) reconstruction, realignment (motion correction), and processing of brain images as soon as they are acquired (typically around 2 seconds); and 3) online feedback presentation related to the activity of brain regions of interest (ROIs) using images (eg, functional maps, thermometer graphs), sounds, or even tactile devices. ${ }^{43}$

Typically, the experiments follow an experimental paradigm encompassing the following sequence: 1) experimental task designed to trigger specific responses (ie, cognitive processes, emotions, symptoms); 2) fMRI localizer run in order to individually map the brain region activated by the experimental task; and 3) rtfMRI run with instructions to upregulate, downregulate, or maintain brain activity as presented in the feedback devices. In cases of clinical trials and group comparisons, the experimental group receives real feedback, while the control group receives sham feedback (most often recorded feedback from another participant or a stochastic-based feedback, such as a random walk). ${ }^{44}$

Even though some technological barriers are still present (eg, real-time correction of movement, signal trends, physiological artifacts; and BOLD signal temporal delay), the number of studies using rtfMRI has been exploding. ${ }^{45}$ To tackle the methodological issues, some computational packages were developed (eg, http://www.brainvoyager.com/ TurboBrainVoyager.html). ${ }^{46-48}$

Recent reviews ${ }^{49}$ highlight that rtfMRI is effective in regulating several cortical and subcortical regions (eg, amygdala, anterior insula, IFG, ACC, superior temporal gyrus, and a diversity of sensorimotor regions), impacting multiple psychological processes (eg, affective states, ${ }^{50}$ evaluation of emotional visual stimuli, auditory attention, or language) and improving several clinical conditions (eg, tinnitus, ${ }^{51}$ nicotine dependence ${ }^{52}$ depression, ${ }^{53}$ psychpathy $\left.{ }^{54}\right)$. Even when aimed at specific brain regions, the effects of rtfMRI are mediated by changes in extended brain network systems. ${ }^{55}$ Additionally, recent studies have also focused on testing the effectiveness 


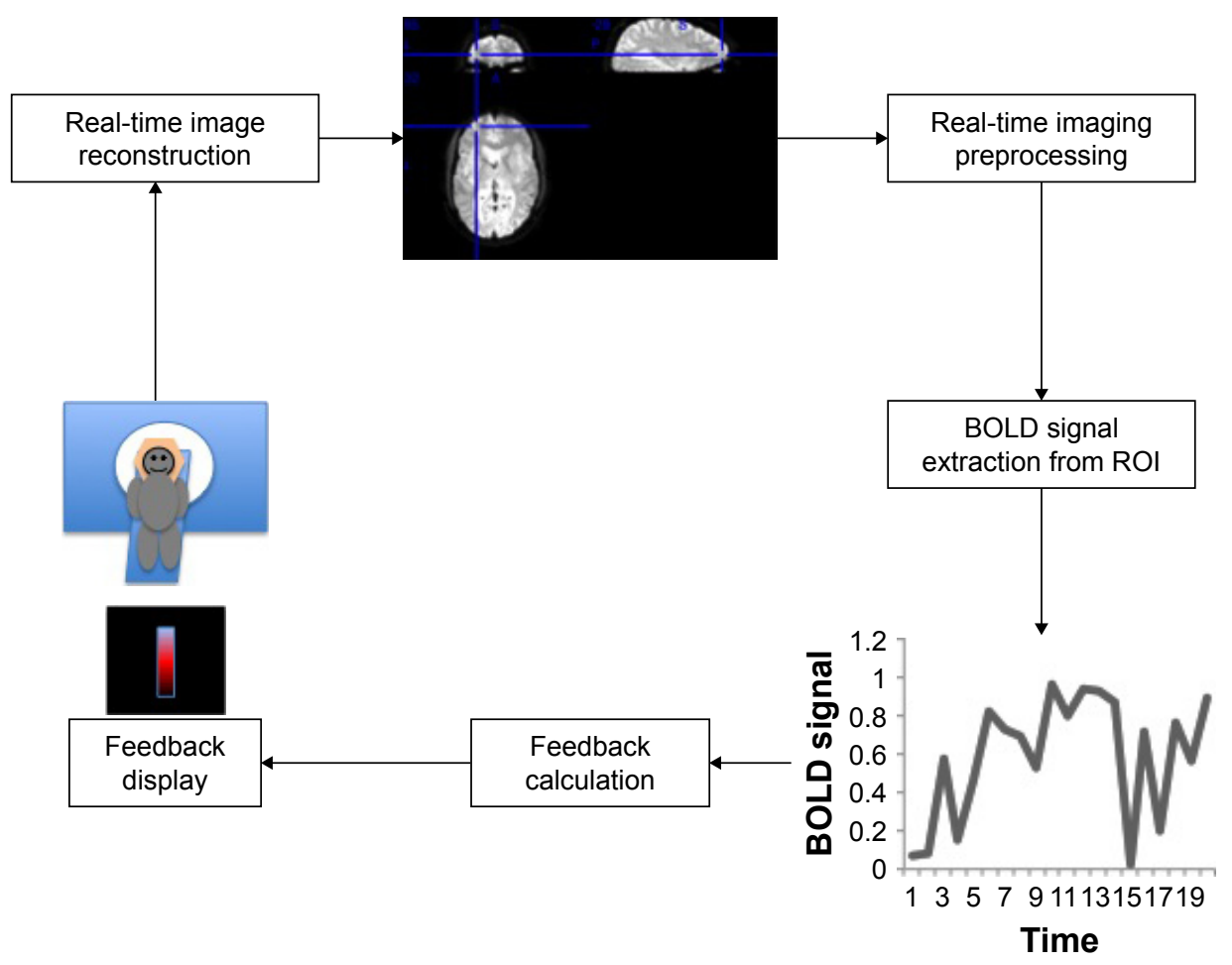

Figure 4 Illustration of the main components of an rtfMRI system.

Abbreviations: rtfMRI, real-time functional magnetic resonance imaging; BOLD, blood oxygenation level-dependent; ROI, region of interest.

of rtfMRI beyond the activation of specific ROIs by providing real-time feedback on the patterns of functional/effective connectivity of certain networks. ${ }^{56-58}$

Another technological advance that moves beyond singleregion activity or functional/effective connectivity modulation is real-time pattern recognition or brain decoding. The basic idea is to apply machine learning methods to classify brain states (output; eg, positive and negative valence) using the BOLD signal from several voxels (even whole brain) as input. Thus, these methods aim to provide feedback based on the recognition patterns of activity across different voxels. Thus, these methods aim to extract functional signatures of certain brain states from a multivariate perspective, that is, by taking into account all input voxels in a joint analysis (multivoxel pattern analysis, MVPA). ${ }^{59}$

Next, we review initial trials testing the use of rtfMRI to target brain regions associated with specific OCD symptoms (eg, contamination) and other mind-brain processes (eg, cognitive - working memory, inhibitory control, emotional defensive and appetitive systems, fear counter-conditioning) found impaired in OCD patients.

\section{Results of studies targeting OCD mind-brain signatures with rtfMRI}

Using rtfMRI with psychiatric disorders is recent with only a few pilots trials available, involving, among others, clinical conditions such as depression, psychopathy, schizophrenia, chronic pain, and smoking. ${ }^{60}$ Overall, the results show that we can train individuals in regulating multiple regions thought to be relevant in each disorder. ${ }^{49}$ Most of these studies are still proof-of-concept trials, lacking the validation rigor required by controlled clinical trials. ${ }^{61}$ This is also true for OCD in which the few pilot studies available contrast with abundant evidence for specific brain alterations found in OCD patients. In order to identify rtfMRI studies in OCD, we started by searching PubMed databases using the following terms: real-time neuroimaging/fMRI/neurofeedback and OCD. Further, we explored reference sections of these articles in order to identify further relevant studies. Only three published studies were found using rtfMRI in OCD or, at least, targeting OCD symptoms.

Next, we review the three published studies using rtfMRI addressing OCD symptoms and then present examples of rtfMRI studies aimed at the modulation of mind-brain processes relevant for OCD as potential avenues for future research (Table 1 presents a summary.).

In a first feasibility study by Buyukturkoglu et al ${ }^{62}$ three OCD patients, with prevalent contamination symptoms, took part in an rtfMRI experiment across 10 days with the aim of decreasing contamination/washing symptoms by downregulating the activity in bilateral anterior insula. The rtfMRI started with a functional localizer run to select, for 
Table I Examples of rtfMRI studies aimed at the modulation of mind-brain processes relevant for OCD

\begin{tabular}{llll}
\hline Domains & Processes & Brain targets & Research trials \\
\hline Symptoms & Contamination & Right anterior insula & Buyukturkoglu et al $^{62}$ \\
& & Orbitofrontal cortex & Scheinost et al ${ }^{64}$ \\
Cognitive regulation & & Sherwood et al ${ }^{70}$ & Nilsen $^{73}$ \\
& Working memory & Left dorsolateral prefrontal cortex & Paret et al $^{75}$ \\
Emotional regulation & Inhibitory control & Right inferior frontal gyrus & Koizumi et al $^{66}$ \\
& Defensive system & Amygdala & Li et al ${ }^{76}$ \\
\hline
\end{tabular}

Note: ${ }^{a}$ rtfMRI studies with healthy samples targeting mind-brain processes relevant for OCD.

Abbreviations: rtfMRI, real-time functional magnetic resonance imaging; $O C D$, obsessive-compulsive disorder.

each patient, ROIs activated by symptom provocative (ie, disgust pictures) and neutral stimuli (ie, landscapes, daily life objects). During baseline blocks, participants were instructed to look at the stimuli without attempting to regulate their emotions. For the downregulation blocks, instructions were to decrease the BOLD signal from the anterior insula using any strategy of their choice. Every time patients were effective in downregulating the BOLD signal, a visual monetary feedback presented contingently. The authors included transfer blocks in each training session (ie, instruction to downregulate the BOLD signal without feedback). Overall, all of the three patients could downregulate the activity on the anterior insula while looking at symptom provocative stimuli. Downregulation of the anterior insula was accompanied, in all participants, with increased activity in middle temporal and left postcentral gyrus, regions associated with the reappraisal or suppression emotional regulation strategies reported by the patients during the rtfMRI downregulation blocks. Finally, in two patients, there was a significant association between brain regulation and clinical changes, as evidenced by their ratings of symptom-provoking pictures and their capability to approach in vivo symptom-provoking stimuli.

The rtfMRI regulation of contamination symptoms in a healthy sample was also addressed in a study by Scheinost et al as part of a research program on the use of rtfMRI with OCD, tic disorder, and posttraumatic stress disorder. ${ }^{63-65}$ This time, the OFC was the brain ROIs. ${ }^{64}$ The authors hypothesize that regulation training of the $\mathrm{OFC}$ (up- and downregulation) would impact the reorganization of relevant brain resting-state networks, contributing to reducing contamination anxiety. Twenty participants with contamination anxiety completed an rtfMRI experiment with four scanning sessions. In the first session, after a resting-state run, a functional localizer (ie, presentation of contamination versus neutral images) was acquired to establish individualized OFC target regions. After being trained and assessed offline in the ability to control anxiety in face of provoking stimuli, participants underwent the rtfMRI training (sessions 2 and 3 ) in which instructions were given to control (upregulating, downregulating, rest) the OFC BOLD line graph signal while being presented contamination or neutral pictures. The rtfMRI runs were interleaved with control runs in which individuals had to apply the strategies they found effective in regulating their emotions but, this time, without feedback. The fourth session included a final assessment and another resting-state acquisition. A control group completed an identical procedure but with sham feedback from a paired participant. The results showed that participants in the experimental conditions learned how to control the activity of the OFC. But, more important, the rtfMRI procedure was effective in producing a connectivity decrease, only in the experimental group, in regions associated with emotional arousal (eg, insula, hippocampus, parahippocampal and entorhinal cortices, right amygdala, brain stem, substantia nigra, temporal pole, superior temporal sulcus, thalamus, fusiform gyrus) and a correlative increased connectivity in core regions for emotional regulation (eg, right lateral PFC). As hypothesized, the effects of brain self-regulation positively impacted contamination anxiety in the experimental group.

In a follow-up study ${ }^{65}$ the authors extend this study to a cohort of OCD patients, testing this time if resting-state brain connectivity would be a good predictor of rtfMRI efficacy in decreasing contamination anxiety symptoms in both healthy and OCD samples. A group of five OCD patients with moderate contamination symptoms followed a similar protocol to the one described above. The authors confirmed the effectiveness of the procedure showing a significant clinical improvement in OCD participants. Interestingly, resting-state connectivity in the OFC before the rtfMRI was a significant predictor of behavioral improvement in contamination symptoms in both healthy and OCD cohorts. As suggested by the authors, it is interesting to note the neurobiological communalities in healthy and clinical population sharing identical symptoms, suggesting that studies with healthy 
population can have important translational implications for OCD patients.

A recent rtfMRI study suggested an alternative method to exposure and response prevention in fear reduction with healthy participants with relevance for OCD treatment. ${ }^{66}$ Using a retinotopic mapping paradigm, the authors were able to classify a red versus green grating patterns with an accuracy of $\sim 72 \%$ (significantly above chance). Then, participants were fear-conditioned to both stimuli (red and green). Finally, during the rtfMRI phase, participants viewed achromatic visual gratings and were asked to use any mental strategy they wanted to increase the diameter of a disc to receive a monetary reward. The size of the diameter (and the reward) was proportional to how similar was their pattern of visual cortex activation for one of the CS+ stimuli (ie, green). Participants could decrease autonomic emotional response and amygdala activation to the green pattern (target condition), suggesting that the training was effective in implicitly reducing the fear via counter-conditioning. Interestingly, none of the subjects reported fear during the training phase, and all of them remained unaware of the association between the disc diameter and the occurrence of the target $\mathrm{CS}+$ representation.

As described, OCD patients exhibit impairments in working memory. ${ }^{67}$ While working memory tasks seem to activate similar brain networks in OCD and healthy controls, OCD patients seem to overcompensate for their working memory impairments with increased dorsolateral (dlPFC) activations. ${ }^{68,69}$ There is now evidence that by learning how to self-regulate, the dlPFC individuals can improve working memory. For example, Sherwood et al ${ }^{70}$ tested if they could train healthy participants to control the left dlPFC response using rtfMRI and the impact of this training on working memory and vigilance tasks. Thirty-two individuals took part in an experiment consisting of five sessions of n-back task plus rtfMRI training (experimental group) or only n-back task and regulation working memory training but without neurofeedback (control group). All participants were assessed before (session 1) and after the training (session 5) with n-back and prospective working memory with vigilant tasks. After a localizer run targeting the left dlPFC, and familiarization with the procedure, participants underwent blocks of rtfMRI training, alternating rest with task conditions with instructions to increase the brain activity associated with working memory using several strategies (eg, recall walking to the laboratory, recent phone call, performing mental math) and trying to upregulate the $\mathrm{dPFC}$ BOLD signal. The results show that the participants from the experimental group learned how to regulate dlPFC activity and increased their performance in prospective working memory and vigilance, but not in the n-back task. Other recent studies have been showing the impact of training regulation of the left dlPFC in other working memories tasks, such as digit span. ${ }^{71}$

OCD patients perform poorly in distinct types of inhibitory control tasks. ${ }^{72}$ As discussed, a core region for the inhibitory control networks - the IFG - is affected in OCD. ${ }^{21}$ An unpublished thesis from Nilsen illustrates the effects of rtfMRI regulation over IFG on response inhibition. ${ }^{73}$ In this study, 30 healthy individuals had to increase (without specific cognitive instructions) either the left or the right opercular region of the IFG across nine blocks (one baseline block plus eight blocks alternating between rest and upregulation training). The authors looked at the effects of the training in two motor inhibition tasks: stop-signal task and Posner cueing task. Even though participants demonstrated a consistent upregulation, no effects were found on the stop-signal task due to the right IFG regulation training. The only significant effect was found with the upregulation of the right IFG on the Posner cueing task, but even here, contrary to the prediction, there was a decreased reorienting efficiency in male participants. Despite this negative result, a recent proof-ofconcept study showed the efficacy of rtfMRI upregulation of right IFG during a stop-signal task with attention-deficit/ hyperactivity disorder patients. ${ }^{74}$

As stated, an emotional imbalance seems to be present in OCD, characterized by an overreactivity to threat (ie, the predominance of a defensive system) contrasting with decreased reactivity to positive safe stimuli (ie, under-dominance of the appetitive system). ${ }^{22}$ Therefore, it may be interesting to explore if the use of rtfMRI strategies targeting regions is associated with the processing of threat. Additionally, it would be important to explore the effectiveness of rtfMRI in upregulating brain networks associated with positive affect.

Paret et $\mathrm{al}^{75}$ tested the effects of rtfMRI regulation of amygdala activity on the modulation of emotional networks. Thirty-two healthy individuals took part in an experiment requiring downregulation of either the amygdala activity (experimental group) or a basal ganglia region (control group) while seeing aversive versus neutral pictures. The rtfMRI took place during only one session, alternating training with rest conditions and ending with a transfer run (similar to the training runs but without feedback). The authors found that, first, the training was effective in downregulating amygdala activity. Second, and most important, the experimental condition was effective in increasing the connectivity between the right amygdala and the ventromedial prefrontal cortex 
(vmPFC) along with increased connectivity between the vmPFC with the visual cortex, hippocampus, and ventrolateral prefrontal cortex. That is, the rtfMRI seemed to impact functional connectivity in a network associated with topdown regulation of the defensive emotional system.

On a different direction, a study by Li et $\mathrm{al}^{76}$ used MVPA and rtfMRI in order to test the possibility of upregulating brain positive emotional networks. More specifically, 23 healthy subjects went through the following five-stage rtfMRI procedure: 1) resting-state run; 2) mental imaging run in which participants were instructed to activate emotions, in alternating blocks, associated with happy, sad, and neutral autobiographical memories; 3 ) the results from this stage were used to train a classification model (happy versus sad emotional states) using MVPA; 4) in stage 4, participants from the experimental group were instructed to try to raise the bar of a graphical "thermometer" (using the activation of autobiographical memories) indicating the activations match with brain regions previously established by the classifier (ie, extent and class of emotion); training blocks alternated with resting blocks; and 5) resting-state run. The results showed that the training was effective in increasing positive emotions (ie, separation between happy and sad neural representations) and that these findings were associated with an increased activity in brain networks associated with emotional processing regions (eg, left insula, left amygdala, ACC, and dorsomedial PFC) and emotional regulation (eg, PFC, temporal cortex, putamen, left caudate, precuneus).

\section{Discussion}

While studies using rtfMRI in OCD are still preliminary, some tentative conclusions can be drawn: 1) Some OCD patients seem to learn how to regulate brain regions (eg, anterior insula; OFC) associated with their symptoms (eg, contamination) with significant clinical impact. 2) Studies with healthy populations have shown that individuals can regulate brain regions associated with cognitive (eg, working memory, inhibitory control) and emotional processes (eg, defensive and appetitive systems) found to be impaired in OCD.

Although promising, these studies are still in "a proof-ofconcept" stage. We need to follow up with controlled clinical trials that should: 1) test rtfMRI in brain regions and networks associated different OCD symptoms (eg, checking, cleaning/ decontamination, counting compulsions; contamination, harm or sexual, symmetry or exactness obsessions); 2) try innovative treatments (eg, counter-conditioning) in patient populations; 3 ) test the translation for OCD of studies done with healthy populations and targeting brain regions associated with OCD cognitive and emotional impairments.

A final note to remark is that rtfMRI is still paving the road to becoming an effective and cost-effective method. ${ }^{77}$ A panel of experts recommended establishing standard procedures for quality control in six domains: neurofeedback signal (eg, artifacts, participant's discriminability signal-tonoise ratio, temporal delay), type of instructions (eg, signal delay, implicit versus explicit), task design (eg, block versus event-related, number of blocks, number of runs per session, number of sessions), transfer (eg, transfer runs without feedback, debriefing), experimental control (eg, sham feedback, feedback from unrelated brain regions, regulation without feedback, inverted feedback), and behavioral effects (eg, motor, cognitive, emotional). ${ }^{78}$ Likewise, several guidelines were put forward to potentiate the clinical translation. ${ }^{79}$ Due to the required environment and high cost of fMRI scanning, clinical translation of rtfMRI is challenging. One possible path is using rtfMRI to establish target regions or functional signatures estimated using fNIRS and EEG, since these two modalities are less exploratory than fMRI. Thus, rtfMRI studies with simultaneous acquisition of $\mathrm{NIRS}^{80}$ or EEG ${ }^{81}$ signals could be helpful to bridge the gap between research and clinical applications.

While researchers are still struggling with these technological and methodological issues, the field is already exploring new avenues such as closed loop ${ }^{82}$ and hyperscanning (eg, two brains $)^{83}$ strategies that may help extend even further the potentialities of rtfMRI.

\section{Disclosure}

The authors report no conflicts of interest in this work.

\section{References}

1. Storch EA, De Nadai AS, Conceição do Rosário M, et al. Defining clinical severity in adults with obsessive-compulsive disorder. Compr Psychiatry. 2015;63:30-35.

2. American Psychiatric Association. Diagnostic and Statistical Manual of Mental Disorders. 5th ed. Arlington, VA: American Psychiatric Publishing; 2013.

3. Stein DJ, Kogan CS, Atmaca M, et al. The classification of obsessivecompulsive and related disorders in the ICD-11. J Affect Disord. 2016; 190:663-674.

4. Abramowitz JS, Jacoby RJ. Obsessive-compulsive disorder in the DSM-5. Clin Psychol Sci Pract. 2014;21(3):221-235.

5. Gonçalves ÓF, Carvalho S, Leite J, Fernandes-Gonçalves A, Carracedo A, Sampaio A. Cognitive and emotional impairments in obsessivecompulsive disorder: evidence from functional brain alterations. Porto Biomed J. 2016;1(3-5):92-105.

6. Milad MR, Rauch SL. Obsessive-compulsive disorder: beyond segregated cortico-striatal pathways. Trends Cogn Sci. 2012;16(1):43-51.

7. de Wit SJ, Alonso P, Schweren L, et al. Multicenter voxel-based morphometry mega-analysis of structural brain scans in obsessivecompulsive disorder. Am J Psychiatry. 2014;171(3):340-349. 
8. Gonçalves ÓF, Marques TR, Lori NF, Sampaio A, Branco MC. Obsessive-compulsive disorder as a visual processing impairment. Med Hypotheses. 2010;74(1):107-109.

9. Gonçalves ÓF, Carvalho S, Leite J, Pocinho F, Relvas J, Fregni F. Obsessive Compulsive Disorder as a functional interhemispheric imbalance at the thalamic level. Med Hypotheses. 2011;77(3):445-447.

10. Rotge JY, Guehl D, Dilharreguy B, et al. Meta-analysis of brain volume changes in obsessive-compulsive disorder. Biol Psychiatry. 2009;65(1):75-83.

11. Hoexter MQ, Miguel EC, Diniz JB, Shavitt RG, Busatto GF, Sato JR. Predicting obsessive-compulsive disorder severity combining neuroimaging and machine learning methods. J Affect Disord. 2013;150(3): 1213-1216.

12. Piras F, Piras F, Chiapponi C, Girardi P, Caltagirone C, Spalletta G. Widespread structural brain changes in OCD: a systematic review of voxel-based morphometry studies. Cortex. 2015;62:89-108.

13. Gonçalves OF, Sousa S, Carvalho S, et al. Alterations of gray and white matter morphology in obsessive compulsive disorder. Psicothema. 2017; 29(1):35-42.

14. van den Heuvel OA, Remijnse PL, Mataix-Cols D, et al. The major symptom dimensions of obsessive-compulsive disorder are mediated by partially distinct neural systems. Brain. 2009;132(Pt 4):853-868.

15. Subirà $\mathrm{M}$, Sato JR, Alonso $\mathrm{P}$, et al. Brain structural correlates of sensory phenomena in patients with obsessive-compulsive disorder. J Psychiatry Neurosci. 2015;40(4):232-240.

16. Spalletta G, Piras F, Fagioli S, Caltagirone C, Piras F. Brain microstructural changes and cognitive correlates in patients with pure obsessive compulsive disorder. Brain Behav. 2014;4(2):261-277.

17. Alonso P, Orbegozo A, Pujol J, et al. Neural correlates of obsessivecompulsive related dysfunctional beliefs. Prog Neuropsychopharmacol Biol Psychiatry. 2013;47:25-32.

18. Gonçalves ÓF, Carvalho S, Leite J, Fernandes-Gonçalves A, Carracedo A, Sampaio A. Gray matter morphological alteration in obsessive compulsive disorder: evidence for an inhibitory control and emotional regulation disorder. Princ Pract Clin Res. 2016;1(1):162-171.

19. Gonçalves ÓF, Carvalho S, Leite J, Fernandes-Gonçalves A, Carracedo A, Sampaio A. Morphometric and connectivity white matter abnormalities in obsessive compulsive disorder. Princ Pract Clin Res. In press 2017.

20. Piras F, Piras F, Caltagirone C, Spalletta G. Brain circuitries of obsessive compulsive disorder: a systematic review and meta-analysis of diffusion tensor imaging studies. Neurosci Biobehav Rev. 2013;37(10 Pt 2): 2856-2877.

21. Gonçalves OF, Sousa S, Maia L, et al. Inferior frontal gyrus white matter abnormalities in obsessive-compulsive disorder. Neuroreport. 2015;26(9):495-500.

22. Gonçalves ÓF, Soares JM, Carvalho S, et al. Brain activation of the defensive and appetitive survival systems in obsessive compulsive disorder. Brain Imaging Behav. 2015;9(2):255-263.

23. Gonçalves ÓF, Soares JM, Carvalho S, et al. Patterns of default mode network deactivation in obsessive compulsive disorder. Sci Rep. 2017; 7:44468.

24. McLaughlin NC, Strong D, Abrantes A, et al. Extinction retention and fear renewal in a lifetime obsessive-compulsive disorder sample. Behav Brain Res. 2015;280:72-77.

25. Diniz JB, Miguel EC, de Oliviera AR, et al. Outlining new frontiers for the comprehension of obsessive-compulsive disorder: a review of its relationship with fear and anxiety. Rev Bras Psiquiatr. 2012;34 (Suppl 1): S81-S92.

26. Basile B, Mancini F, Macaluso E, Caltagirone C, Bozzali M. Abnormal processing of deontological guilt in obsessive-compulsive disorder. Brain Struct Funct. 2014;219(4):1321-1331.

27. Morein-Zamir S, Voon V, Dodds CM, et al. Divergent subcortical activity for distinct executive functions: stopping and shifting in obsessive compulsive disorder. Psychol Med. 2016;46(4):829-840.

28. Gu BM, Park JY, Kang DH, et al. Neural correlates of cognitive inflexibility during task-switching in obsessive-compulsive disorder. Brain. 2008;131(Pt 1):155-164.
29. Diwadkar VA, Burgess A, Hong E, et al. Dysfunctional activation and brain network profiles in youth with obsessive-compulsive disorder: a focus on the dorsal anterior cingulate during working memory. Front Hum Neurosci. 2015;9:149.

30. Figee M, Vink M, de Geus F, et al. Dysfunctional reward circuitry in obsessive-compulsive disorder. Biol Psychiatry. 2011;69(9):867-874.

31. Liu X, Cui H, Wei Q, et al. Electroconvulsive therapy on severe obsessive-compulsive disorder comorbid depressive symptoms. Psychiatry Investig. 2014;11(2):210-213.

32. Berlim MT, Neufeld NH, Van den Eynde F. Repetitive transcranial magnetic stimulation (rTMS) for obsessive-compulsive disorder (OCD): an exploratory meta-analysis of randomized and sham-controlled trials. J Psychiatr Res. 2013;47(8):999-1006.

33. Bation R, Poulet E, Haesebaert F, Saoud M, Brunelin J. Transcranial direct current stimulation in treatment-resistant obsessive-compulsive disorder: an open-label pilot study. Prog Neuropsychopharmacol Biol Psychiatry. 2016;65:153-157.

34. Romanelli RJ, Wu FM, Gamba R, Mojtabai R, Segal JB. Behavioral therapy and serotonin reuptake inhibitor pharmacotherapy in the treatment of obsessive-compulsive disorder: a systematic review and meta-analysis of head-to-head randomized controlled trials. Depress Anxiety. 2014;31(8):641-652.

35. Gonçalves ÓF, Boggio PS. Neuromodulação Autorregulatória: Princípios E Prática [Self-regulatory Neuromodulation: Foundations and Practice]. São Paulo: Pearson; 2016. Portuguese.

36. Fetz EE. Operant conditioning of cortical unit activity. Science. 1969; 163(3870):955-958.

37. Pascual-Marqui RD, Lehmann D, Koukkou M, et al. Assessing interactions in the brain with exact low-resolution electromagnetic tomography. Philos Trans A Math Phys Eng Sci. 2011;369(1952):3768-3784.

38. Cannon R, Lubar J, Congedo M, Thorton K, Towler K, Hutchens T. The effects of neurofeedback training in the cognitive division of the anterior cingulate gyrus. Int J Neurosci. 2007;117(3):337-357.

39. Kopřivová J, Congedo M, Raszka M, Praško J, Brunovský M, Horáček J. Prediction of treatment response and the effect of independent component neurofeedback in obsessive-compulsive disorder: a randomized, sham-controlled, double-blind study. Neuropsychobiology. 2013;67(4): $210-223$.

40. Logothetis NK, Pauls J, Augath M, Trinath T, Oeltermann A. Neurophysiological investigation of the basis of the fMRI signal. Nature. 2001;412(6843):150-157.

41. Thibault RT, Lifshitz M, Raz A. The self-regulating brain and neurofeedback: experimental science and clinical promise. Cortex. 2016;74: 247-261.

42. Kober SE, Wood G, Kurzmann J, et al. Near-infrared spectroscopy based neurofeedback training increases specific motor imagery related cortical activation compared to sham feedback. Biol Psychol. 2014;95:21-30.

43. Birbaumer N, Ruiz S, Sitaram R. Learned regulation of brain metabolism. Trends Cogn Sci. 2013;17(6):295-302.

44. Sokunbi MO. Feedback of real-time fMRI signals: from concepts and principles to therapeutic interventions. Magn Reson Imaging. 2017;35: $117-124$.

45. Thibault RT, Lifshitz M, Birbaumer N, Raz A. Neurofeedback, selfregulation, and brain imaging: clinical science and fad in the service of mental disorders. Psychother Psychosom. 2015;84(4):193-207.

46. Sato JR, Basilio R, Paiva FF, et al. Real-time fMRI pattern decoding and neurofeedback using FRIEND: an FSL-integrated BCI toolbox. PLoS One. 2013;8(12):e81658.

47. Rana M, Gupta N, Dalboni Da Rocha JL, Lee S, Sitaram R. A toolbox for real-time subject-independent and subject-dependent classification of brain states from fMRI signals. Front Neurosci. 2013;7:170.

48. Basilio R, Garrido GJ, Sato JR, et al. Friend engine framework: a real time neuro feedback client-server system for neuro imaging studies. Front Behav Neurosci. 2015;9:3.

49. Ruiz S, Buyukturkoglu K, Rana M, Birbaumer N, Sitaram R. Real-time fMRI brain computer interfaces: self-regulation of single brain regions to networks. Biol Psychol. 2014;95:4-20. 
50. Moll J, Weingartner JH, Bado P, et al. Voluntary enhancement of neural signatures of affiliative emotion using fMRI neurofeedback. PLoS One. 2014;9(5):e97343.

51. Emmert K, Kopel R, Koush Y, et al. Continuous vs. intermittent neurofeedback to regulate auditory cortex activity of tinnitus patients using real-time fMRI - a pilot study. Neuroimage Clin. 2017;14:97-104.

52. Hartwell KJ, Hanlon CA, Li X, et al. Individualized real-time fMRI neurofeedback to attenuate craving in nicotine-dependent smokers. J Psychiatry Neurosci. 2016;41(1):48-55.

53. Young KD, Siegle GJ, Zotev V, et al. Randomized clinical trial of real-time fMRI amygdala neurofeedback for major depressive disorder: effects on symptoms and autobiographical memory recall. Am J Psychiatry. 2017:appi.ajp.2017.16060637.

54. Sitaram R, Caria A, Veit R, Gaber T, Ruiz S, Birbaumer N. Volitional control of the anterior insula in criminal psychopaths using real-time fMRI neurofeedback: a pilot study. Front Behav Neurosci. 2014;8:344.

55. Haller S, Kopel R, Jhooti P, et al. Dynamic reconfiguration of human brain functional networks through neurofeedback. Neuroimage. 2013; $81: 243-252$.

56. Koush Y, Joao M, Robineau F, et al. Connectivity-based neurofeedback: dynamic causal modeling for real-time fMRI. Neuroimage. 2013; $81: 422-430$.

57. Kadosh KC, Luo Q, de Burca C, et al. Using real-time fMRI to influence effective connectivity in the developing emotion regulation network. Neuroimage. 2016;125:616-626.

58. Koush Y, Meskaldji D-E, Pichon S, et al. Learning control over emotion networks through connectivity-based neurofeedback. Cereb Cortex. 2017;27(2):1193-1202.

59. LaConte SM. Decoding fMRI brain states in real-time. Neuroimage. 2011;56(2):440-454.

60. Brühl AB. Making sense of real-time functional magnetic resonance imaging (rtfMRI) and rtfMRI neurofeedback. Int J Neuropsychopharmacol. 2015;18(6):pyv020.

61. Sitaram R, Ros T, Stoeckel L, et al. Closed-loop brain training: the science of neurofeedback. Nat Rev Neurosci. 2017;18(2):86-100.

62. Buyukturkoglu K, Roettgers H, Sommer J, et al. Self-regulation of anterior insula with real-time fMRI and its behavioral effects in obsessive-compulsive disorder: a feasibility study. PLoS One. 2015; 10(8):e0135872.

63. Hampson M, Stoica T, Saksa J, et al. Real-time fMRI biofeedback targeting the orbitofrontal cortex for contamination anxiety. J Vis Exp. 2012;59(59):e3535.

64. Scheinost D, Stoica T, Saksa J, et al. Orbitofrontal cortex neurofeedback produces lasting changes in contamination anxiety and resting-state connectivity. Transl Psychiatry. 2013;3:e250.

65. Scheinost D, Stoica T, Wasylink S, et al. Resting state functional connectivity predicts neurofeedback response. Front Behav Neurosci. 2014;8:338

66. Koizumi A, Amano K, Cortese A, et al. Fear reduction without fear through reinforcement of neural activity that bypasses conscious exposure. Nat Hum Behav. 2016;1:6.
67. Jaafari N, Rigalleau F, Rachid F, et al. A critical review of the contribution of eye movement recordings to the neuropsychology of obsessive compulsive disorder. Acta Psychiatr Scand. 2011;124(2):87-101.

68. Henseler I, Gruber O, Kraft S, Krick C, Reith W, Falkai P. Compensatory hyperactivations as markers of latent working memory dysfunctions in patients with obsessive-compulsive disorder: an fMRI study. J Psychiatry Neurosci. 2008;33(3):209-215.

69. Nakao T, Nakagawa A, Yoshiura T, et al. Duration effect of obsessivecompulsive disorder on cognitive function: a functional MRI study. Depress Anxiety. 2009;26(9):814-823.

70. Sherwood MS, Weisend MP, Kane JH, Parker JG. Combining real-time fMRI neurofeedback training of the DLPFC with N-back practice results in neuroplastic effects confined to the neurofeedback target region. Front Behav Neurosci. 2016;10:138.

71. Zhang G, Yao L, Zhang H, Long Z, Zhao X. Improved working memory performance through self-regulation of dorsal lateral prefrontal cortex activation using real-time fMRI. PLoS One. 2013;8(8):e73735.

72. van Velzen LS, Vriend C, de Wit SJ, van den Heuvel OA. Response inhibition and interference control in obsessive-compulsive spectrum disorders. Front Hum Neurosci. 2014;8:419.

73. Nilsen AS. Right Inferior Frontal Gyrus and Motor Response Inhibition: A Real-Time fMRI Neurofeedback Study [master's thesis]. Oslo: University of Oslo; 2014.

74. Alegria AA, Wulff M, Brinson H, et al. Real-time fMRI neurofeedback in adolescents with attention deficit hyperactivity disorder. Hum Brain Mapp. 2017;38(6):3190-3209.

75. Paret C, Ruf M, Fungisai M, et al. fMRI neurofeedback of amygdala response to aversive stimuli enhances prefrontal-limbic brain connectivity. Neuroimage. 2016;125:182-188.

76. Li Z, Tong L, Wang L, et al. Self-regulating positive emotion networks by feedback of multiple emotional brain states using real-time fMRI. Exp Brain Res. 2016;234(12):3575-3586.

77. Thibault RT, Raz A. When can neurofeedback join the clinical armamentarium? Lancet Psychiatry. 2016;3(6):497-498.

78. Sulzer J, Haller S, Scharnowski F, et al. Real-time fMRI neurofeedback: progress and challenges. Neuroimage. 2013;76:386-399.

79. Stoeckel LE, Garrison KA, Ghosh S, et al. Optimizing real time fMRI neurofeedback for therapeutic discovery and development. Neuroimage Clin. 2014;5:245-255.

80. Cui X, Bray S, Bryant DM, Glover GH, Reiss AL. A quantitative comparison of NIRS and fMRI across multiple cognitive tasks. Neuroimage. 2011;54(4):2808-2821.

81. Rosenkranz K, Lemieux L. Present and future of simultaneous EEGfMRI. MAGMA. 2010;23(5-6):309-316.

82. deBettencourt MT, Cohen JD, Lee RF, Norman KA, Turk-Browne NB. Closed-loop training of attention with real-time brain imaging. Nat Neurosci. 2015;18(3):470-475.

83. Babiloni F, Astolfi L. Social neuroscience and hyperscanning techniques: past, present and future. Neurosci Biobehav Rev. 2014;44:76-93.
Neuropsychiatric Disease and Treatment

\section{Publish your work in this journal}

Neuropsychiatric Disease and Treatment is an international, peerreviewed journal of clinical therapeutics and pharmacology focusing on concise rapid reporting of clinical or pre-clinical studies on a range of neuropsychiatric and neurological disorders. This journal is indexed on PubMed Central, the 'PsycINFO' database and CAS,
Dovepress

and is the official journal of The International Neuropsychiatric Association (INA). The manuscript management system is completely online and includes a very quick and fair peer-review system, which is all easy to use. Visit http://www.dovepress.com/testimonials.php to read real quotes from published authors. 